\title{
Perturbation to Noether Symmetry and Noether Adiabatic Invariants of Discrete Difference Variational Hamilton Systems
}

\author{
M.-J. ZHANG ${ }^{a, b, *}$ AND J.-H. FANG ${ }^{c}$ \\ ${ }^{a}$ Purple Mountain Observatory, Chinese Academy of Sciences, Nanjing 210008, China \\ ${ }^{b}$ Key Laboratory of Space Object and Debris Observation, PMO, CAS, Nanjing 210008, China \\ ${ }^{c}$ College of Science, China University of Petroleum, Qingdao 266555, China
}

(Received April 15, 2011; in final form March 2, 2012)

\begin{abstract}
The perturbation to the Noether symmetry and the Noether adiabatic invariants of discrete difference variational Hamilton systems are investigated. The discrete the Noether exact invariant induced directly by the the Noether symmetry of the system without perturbation is given. The concept of discrete high-order adiabatic invariant is presented, the criterion of the perturbation to the Noether symmetry is established, and the discrete the Noether adiabatic invariant induced directly by the perturbation to the Noether symmetry is obtained. Lastly, an example is discussed to illustrate the application of the results.
\end{abstract}

PACS: $02.20 . \mathrm{Sv}, 11.30 .-\mathrm{j}, 45.05 .+\mathrm{x}$

\section{Introduction}

The research on symmetries and conserved quantities of a mechanical system possesses important mathematical and physical significance, and is also an important development direction in analytical mechanics. In Refs. [1, 2] symmetries and conserved quantities of constrained mechanical systems have been thoroughly investigated. Moreover, symmetries and conserved quantities of more general systems (optimal control problems) are also studied in depth [3-5]. Recently, researches on symmetries and conserved quantities have been extended to a general time scale (including, as particular cases, both discrete and continuous settings). Levi et al. [6-8] first extended the Lie symmetry to discrete systems; Dorodnitsyn [9] adapted the Noether's theory to discrete Lagrangian system; Torres et al. [10-12] studied the Noether symmetry theorems for an arbitrary time scale; Shi et al. [13, 14] investigated Lie symmetry and the Noether conserved quantities (or exact invariants) of discrete non-conservative mechanical systems and discrete difference variational Hamilton systems without perturbations; based upon the property of the discrete models entirely inheriting the symmetry of the continuous systems, Fu et al. [15] presented the Hojman conserved quantities and Lie symmetries of discrete mechanico-electrical coupling systems by the Lie groups of transformations of continuous systems.

As we know, even a tiny disturbance acting on the mechanical systems, that we can call a perturbation, may influence the original symmetries and conserved quantities of mechanical systems. Pioneered in this area, Burgers [16] proposed adiabatic invariants for a special kind of the Hamilton systems. Perturbation to symmetries and

* corresponding author; e-mail: mjzhang@pmo.ac.cn adiabatic invariants play a very important role in the research on the quasi-integrability of mechanical systems. A classical adiabatic invariant is a certain physical quantity that changes more slowly than some slowly-varying parameter of the system [17]. In fact, the parameter varying very slowly is equivalent to the action of a small disturbance. At present, studies in this field have become very active, and many important results have been obtained [18-23]. But all these studies have focused on perturbation to symmetries and adiabatic invariants of the continuous mechanical systems. Recently, Zhang et al. [24] presented the concept of discrete high-order adiabatic invariant, and studied the perturbation to the the Noether symmetry and the Noether adiabatic invariant of the general discrete holonomic system. Wang and Zhu [25] further discussed perturbation to symmetry and adiabatic invariants of general discrete holonomic dynamical systems on a uniform lattice. However, perturbation to symmetries and adiabatic invariants of the discrete Hamilton systems has never been studied so far.

In this paper, based on the concept of discrete high-order adiabatic invariant, the perturbation to the Noether symmetry and the Noether adiabatic invariants of discrete difference variational Hamilton systems are studied. The discrete the Noether exact invariant induced directly by the the Noether symmetry of the system without perturbation is given. The criterion of the perturbation to the Noether symmetry is established, and the discrete the Noether adiabatic invariant induced directly by the perturbation to the Noether symmetry is obtained. Meanwhile, an example is discussed to illustrate the application of the results.

\section{2. the Noether symmetry and discrete the} Noether exact invariant

For brevity of notation, we consider a one-dimensional discrete difference variational Hamil- 
ton system. The Hamiltonian of the system is $H_{\mathrm{d}}\left(t_{k}, t_{k+1}, q_{k}, q_{k+1}, p_{k}, p_{k+1}\right)(k=0,1, \ldots, N-1), t_{k}$, $q_{k}$, and $p_{k}$ are discrete time, discrete generalized coordinates, and discrete generalized momenta, respectively.

The discrete equations of motion of the system can be written as [14]:

$$
\begin{aligned}
& \frac{1}{2}\left(p_{k-1}-p_{k+1}\right)-D_{3} H_{\mathrm{d}}\left(\varphi_{k}\right)\left(t_{k+1}-t_{k}\right) \\
& \quad-D_{4} H_{\mathrm{d}}\left(\varphi_{k-1}\right)\left(t_{k}-t_{k-1}\right)=0, \\
& \frac{1}{2}\left(q_{k+1}-q_{k-1}\right)-D_{5} H_{\mathrm{d}}\left(\varphi_{k}\right)\left(t_{k+1}-t_{k}\right) \\
& \quad-D_{6} H_{\mathrm{d}}\left(\varphi_{k-1}\right)\left(t_{k}-t_{k-1}\right)=0, \\
& \quad H_{\mathrm{d}}\left(\varphi_{k}\right)-H_{\mathrm{d}}\left(\varphi_{k-1}\right)-D_{1} H_{\mathrm{d}}\left(\varphi_{k}\right)\left(t_{k+1}-t_{k}\right) \\
& \quad-D_{2} H_{\mathrm{d}}\left(\varphi_{k-1}\right)\left(t_{k}-t_{k-1}\right)=0,
\end{aligned}
$$

where $D_{j}$ is the partial derivative of the discrete function with respect to the argument $j$ and $\varphi_{k}=\left(t_{k}, t_{k+1}, q_{k}, q_{k+1}, p_{k}, p_{k+1}\right)$ represents the discrete sequence.

We introduce the infinitesimal transformations with respect to discrete time $t_{k}$, discrete generalized coordinates $q_{k}$, and discrete generalized momenta $p_{k}$ as

$$
\begin{aligned}
& t_{k}^{*}=t_{k}+\Delta t_{k}=t_{k}+\varepsilon \tau_{k}^{0}\left(t_{k}, q_{k}, p_{k}\right), \\
& q_{k}^{*}=q_{k}+\Delta q_{k}=q_{k}+\varepsilon \xi_{k}^{0}\left(t_{k}, q_{k}, p_{k}\right), \\
& p_{k}^{*}=p_{k}+\Delta p_{k}=q_{k}+\varepsilon \eta_{k}^{0}\left(t_{k}, q_{k}, p_{k}\right),
\end{aligned}
$$

where $\varepsilon$ is an infinitesimal group parameter, $\tau_{k}^{0}, \xi_{k}^{0}$ and $\eta_{k}^{0}$ are the discrete infinitesimal generators. The vector field of generators is

$$
X_{0, \mathrm{~d}}^{(0)}=\tau_{k}^{0} \frac{\partial}{\partial t_{k}}+\xi_{k}^{0} \frac{\partial}{\partial q_{k}}+\eta_{k}^{0} \frac{\partial}{\partial p_{k}},
$$

which can be prolonged to the two-point scheme

$$
\begin{aligned}
& X_{0, \mathrm{~d}}^{(1)}=X_{0, \mathrm{~d}}^{(0)}+\tau_{k+1}^{0} \frac{\partial}{\partial t_{k+1}}+\xi_{k+1}^{0} \frac{\partial}{\partial q_{k+1}} \\
& \quad+\eta_{k+1}^{0} \frac{\partial}{\partial p_{k+1}} .
\end{aligned}
$$

The recursive and derivative operators of discrete systems for any discrete variable or function are represented as

$$
\begin{aligned}
& R_{ \pm} f\left(z_{k}\right)=f\left(z_{k \pm 1}\right), \\
& D_{\mathrm{d}} f\left(z_{k}\right)=\frac{R_{+} f\left(z_{k}\right)-f\left(z_{k}\right)}{t_{k+1}-t_{k}} .
\end{aligned}
$$

The the Noether symmetry is an invariance of the Hamiltonian action functional under the infinitesimal transformations. Then the requirement of the the Noether symmetry of the discrete difference variational Hamilton system gives

$$
\begin{aligned}
& H_{\mathrm{d}}\left(\varphi_{k}\right) D_{\mathrm{d}}\left(\tau_{k}^{0}\right)+X_{0, \mathrm{~d}}^{(1)}\left[H_{\mathrm{d}}\left(\varphi_{k}\right)\right]-\frac{1}{2}\left(p_{k+1}+p_{k}\right) D_{\mathrm{d}}\left(\xi_{k}^{0}\right) \\
& \quad+\frac{1}{2}\left(q_{k+1}+q_{k}\right) D_{\mathrm{d}}\left(\eta_{k}^{0}\right)+D_{\mathrm{d}}\left(G_{N k}^{0}\right)=0,
\end{aligned}
$$

where $G_{N k}^{0}=G_{N k}^{0}\left(t_{k}, q_{k}, p_{k}\right)$ is a discrete gauge function.

Criterion 1. If a discrete gauge function $G_{N k}^{0}$ exists such that the infinitesimal generators $\tau_{k}^{0}, \xi_{k}^{0}$ and $\eta_{k}^{0}$ satisfy Eq. (9), the invariance is the Noether symmetry of the discrete difference variational Hamilton system (1), and (3).

Equation (9) is called the discrete the Noether identity of the discrete difference variational Hamilton system (1), (2) and (3).

Theorem $1[14]$. For the discrete difference variational Hamilton system (1), (2) and (3), if the infinitesimal generators $\tau_{k}^{0}, \xi_{k}^{0}$ and $\eta_{k}^{0}$ of the the Noether symmetry and discrete gauge function $G_{N k}^{0}$ satisfy the discrete the Noether identity (9), the the Noether symmetry of the system can induce the discrete the Noether exact invariant

$$
\begin{aligned}
& I_{\mathrm{N} 0, \mathrm{~d}}=\tau_{k}^{0}\left(t_{k}-t_{k-1}\right) D_{2}\left[R_{-} H_{\mathrm{d}}\left(\varphi_{k}\right)\right] \\
& \quad+\xi_{k}^{0}\left(t_{k}-t_{k-1}\right) D_{4}\left[R_{-} H_{\mathrm{d}}\left(\varphi_{k}\right)\right] \\
& \quad+\eta_{k}^{0}\left(t_{k}-t_{k-1}\right) D_{6}\left[R_{-} H_{\mathrm{d}}\left(\varphi_{k}\right)\right]+\tau_{k}^{0} R_{-} H_{\mathrm{d}}\left(\varphi_{k}\right) \\
& \quad-\frac{1}{2}\left(p_{k-1}+p_{k}\right) \xi_{k}^{0}+\frac{1}{2}\left(q_{k-1}+q_{k}\right) \eta_{k}^{0}+G_{N k}^{0}=\text { const. }
\end{aligned}
$$

\section{Perturbation to the Noether symmetry and discrete the Noether adiabatic invariant}

Small force acting on the discrete difference variational Hamilton system may result in a small change in its symmetries, i.e. perturbation to symmetries. The conserved quantity associated with the symmetries, under a corresponding change, is an adiabatic invariant. In analytical mechanics, we study perturbation to symmetries and adiabatic invariants of mechanical systems based on the concept of high-order adiabatic invariant. According to the concept of adiabatic invariant [17], for the discrete difference variational Hamilton system, we have

Definition. If $L_{z, \mathrm{~d}}\left(t_{k}, t_{k+1}, q_{k}, q_{k+1}, p_{k}, p_{k+1}, \varepsilon\right)$ is a physical quantity including $\varepsilon$ in which the highest power is $z$ in a discrete difference variational Hamilton system, and its derivative with respect to discrete time $t_{k}$ is directly proportional to $\varepsilon^{z+1}, I_{z, \mathrm{~d}}$ is called $a z$-th order adiabatic invariant of the discrete difference variational Hamilton system. Definition. If $L_{z, \mathrm{~d}}\left(t_{k}, t_{k+1}, q_{k}, q_{k+1}, p_{k}, p_{k+1}, \varepsilon\right)$ is a physical quantity including $\varepsilon$ in which the highest power is $z$ in a discrete difference variational Hamilton system, and its derivative with respect to discrete time $t_{k}$ is directly proportional to $\varepsilon^{z+1}, I_{z, \mathrm{~d}}$ is called a $z$-th order adiabatic invariant of the discrete difference variational Hamilton system.

Suppose the discrete difference variational Hamilton system (1), (2) and (3) is perturbed by small quantity $\varepsilon W_{\mathrm{d}}\left(t_{k}, t_{k+1}, q_{k}, q_{k+1}, p_{k}, p_{k+1}\right)=\varepsilon W_{\mathrm{d}}\left(\varphi_{k}\right)$, then we have the following total variation of corresponding discrete Hamiltonian action functional:

$$
\begin{gathered}
\Delta S_{\mathrm{d}}+\sum_{k=0}^{N-1} \varepsilon W_{\mathrm{d}}\left(\varphi_{k}\right)\left(t_{k+1}-t_{k}\right) \Delta q_{k} \\
-\sum_{k=0}^{N-1} \varepsilon W_{\mathrm{d}}\left(\varphi_{k}\right)\left(q_{k+1}-q_{k}\right) \Delta t_{k}=0,
\end{gathered}
$$

where $\Delta$ is a total variation symbol, and 


$$
\begin{aligned}
S_{\mathrm{d}} & =\sum_{k=0}^{N-1} \frac{1}{2}\left(p_{k+1}+p_{k}\right)\left(q_{k+1}-q_{k}\right) \\
& -\sum_{k=0}^{N-1} H_{\mathrm{d}}\left(t_{k}, t_{k+1}, q_{k}, q_{k+1}, p_{k}, p_{k+1}\right)\left(t_{k+1}-t_{k}\right) .
\end{aligned}
$$

From (11), using the similar deduction in [14], we obtain the discrete equations of motion of the discrete difference variational Hamilton system perturbed by small quantity $\varepsilon W_{\mathrm{d}}\left(\varphi_{k}\right)$ :

$$
\begin{aligned}
& \frac{1}{2}\left(p_{k-1}-p_{k+1}\right)-D_{3} H_{\mathrm{d}}\left(\varphi_{k}\right)\left(t_{k+1}-t_{k}\right) \\
& \quad-D_{4} H_{\mathrm{d}}\left(\varphi_{k-1}\right)\left(t_{k}-t_{k-1}\right) \\
& \quad+\varepsilon W_{\mathrm{d}}\left(\varphi_{k}\right)\left(t_{k+1}-t_{k}\right)=0, \\
& \frac{1}{2}\left(q_{k+1}-q_{k-1}\right)-D_{5} H_{\mathrm{d}}\left(\varphi_{k}\right)\left(t_{k+1}-t_{k}\right) \\
& \quad-D_{6} H_{\mathrm{d}}\left(\varphi_{k-1}\right)\left(t_{k}-t_{k-1}\right)=0, \\
& H_{\mathrm{d}}\left(\varphi_{k}\right)-H_{\mathrm{d}}\left(\varphi_{k-1}\right)-D_{1} H_{\mathrm{d}}\left(\varphi_{k}\right)\left(t_{k+1}-t_{k}\right) \\
& \quad-D_{2} H_{\mathrm{d}}\left(\varphi_{k-1}\right)\left(t_{k}-t_{k-1}\right) \\
& \quad-\varepsilon W_{\mathrm{d}}\left(\varphi_{k}\right)\left(q_{k+1}-q_{k}\right)=0 .
\end{aligned}
$$

Because of the action of $\varepsilon W_{\mathrm{d}}\left(\varphi_{k}\right)$, the original symmetries and invariants of the system may vary. Assume that the variation is a small perturbation based on the symmetrical transformations of the system without perturbation, and $\tau_{k}\left(t_{k}, q_{k}, p_{k}\right), \xi_{k}\left(t_{k}, q_{k}, p_{k}\right)$ and $\eta_{k}\left(t_{k}, q_{k}, p_{k}\right)$ express the generators of infinitesimal transformations after being perturbed, then

$$
\begin{aligned}
\tau_{k} & =\tau_{k}^{0}+\varepsilon \tau_{k}^{1}+\varepsilon^{2} \tau_{k}^{2}+\ldots, \\
\xi_{k} & =\xi_{k}^{0}+\varepsilon \xi_{k}^{1}+\varepsilon^{2} \xi_{k}^{2}+\ldots, \\
\eta_{k} & =\eta_{k}^{0}+\varepsilon \eta_{k}^{1}+\varepsilon^{2} \eta_{k}^{2}+\ldots
\end{aligned}
$$

The infinitesimal transformations become

$$
\begin{aligned}
& t_{k}^{*}=t_{k}+\Delta t_{k}=t_{k}+\varepsilon \tau_{k}\left(t_{k}, q_{k}, p_{k}\right), \\
& q_{k}^{*}=q_{k}+\Delta q_{k}=q_{k}+\varepsilon \xi_{k}\left(t_{k}, q_{k}, p_{k}\right), \\
& p_{k}^{*}=p_{k}+\Delta p_{k}=p_{k}+\varepsilon \eta_{k}\left(t_{k}, q_{k}, p_{k}\right) .
\end{aligned}
$$

According to the the Noether symmetry theory, the the Noether identity of the discrete difference variational Hamilton system perturbed by small quantity $\varepsilon W_{\mathrm{d}}\left(\varphi_{k}\right)$ becomes

$$
\begin{aligned}
& H_{\mathrm{d}}\left(\varphi_{k}\right) D_{\mathrm{d}}\left(\tau_{k}\right)+X_{\mathrm{d}}^{(1)}\left[H_{\mathrm{d}}\left(\varphi_{k}\right)\right]-\frac{1}{2}\left(p_{k+1}+p_{k}\right) D_{\mathrm{d}}\left(\xi_{k}\right) \\
& \quad+\frac{1}{2}\left(q_{k+1}+q_{k}\right) D_{\mathrm{d}}\left(\eta_{k}\right)-\varepsilon W_{\mathrm{d}}\left(\varphi_{k}\right)\left[\xi_{k}-D_{\mathrm{d}}\left(q_{k}\right) \tau_{k}\right] \\
& \quad+D_{\mathrm{d}}\left(G_{N k}\right)=0,
\end{aligned}
$$

where

$$
\begin{aligned}
& X_{\mathrm{d}}^{(1)}=\tau_{k} \frac{\partial}{\partial t_{k}}+\xi_{k} \frac{\partial}{\partial q_{k}}+\eta_{k} \frac{\partial}{\partial p_{k}}+\tau_{k+1} \frac{\partial}{\partial t_{k+1}} \\
& \quad+\xi_{k+1} \frac{\partial}{\partial q_{k+1}}+\eta_{k+1} \frac{\partial}{\partial p_{k+1}},
\end{aligned}
$$

and $G_{N k}=G_{N k}\left(t_{k}, q_{k}, p_{k}\right)$ is a gauge function. After being perturbed, the gauge function comes into

$$
G_{N k}=G_{N k}^{0}+\varepsilon G_{N k}^{1}+\varepsilon^{2} G_{N k}^{2}+\ldots
$$

Substituting (16) into (19), we have

$$
X_{\mathrm{d}}^{(1)}=\varepsilon^{m} X_{\mathrm{m}, \mathrm{d}}^{(1)} \quad(m=0,1, \ldots, z),
$$

where

$$
\begin{aligned}
& X_{\mathrm{m}, \mathrm{d}}^{(1)}=\tau_{k}^{m} \frac{\partial}{\partial t_{k}}+\xi_{k}^{m} \frac{\partial}{\partial q_{k}}+\eta_{k}^{m} \frac{\partial}{\partial p_{k}}+\tau_{k+1}^{m} \frac{\partial}{\partial t_{k+1}} \\
& \quad+\xi_{k+1}^{m} \frac{\partial}{\partial q_{k+1}}+\eta_{k+1}^{m} \frac{\partial}{\partial p_{k+1}} .
\end{aligned}
$$

Substituting (16) into (18), noticing (19)-(22), and making the coefficients of $\varepsilon^{m}$ equal, we obtain

$$
\begin{aligned}
& H_{\mathrm{d}}\left(\varphi_{k}\right) D_{\mathrm{d}}\left(\tau_{k}^{m}\right)+X_{\mathrm{m}, \mathrm{d}}^{(1)}\left[H_{\mathrm{d}}\left(\varphi_{k}\right)\right] \\
& \quad-\frac{1}{2}\left(p_{k+1}+p_{k}\right) D_{\mathrm{d}}\left(\xi_{k}^{m}\right)+\frac{1}{2}\left(q_{k+1}+q_{k}\right) D_{\mathrm{d}}\left(\eta_{k}^{m}\right) \\
& \quad-W_{\mathrm{d}}\left(\varphi_{k}\right)\left[\xi_{k}^{m-1}-D_{\mathrm{d}}\left(q_{k}\right) \tau_{k}^{m-1}\right]+D_{\mathrm{d}}\left(G_{N k}^{m}\right)=0,
\end{aligned}
$$

when $m=0$, we note that $\tau_{k}^{m-1}=\xi_{k}^{m-1}=\eta_{k}^{m-1}=0$ holds, then Eq. (23) turns into Eq. (9).

Criterion 2. For the discrete difference variational Hamilton system (1), (2) and (3) perturbed by small quantity $\varepsilon W_{\mathrm{d}}\left(\varphi_{k}\right)$, if the discrete gauge function $G_{N k}^{m}$ exists such that the infinitesimal generators $\tau_{k}^{m}, \xi_{k}^{m}$ and $\eta_{k}^{m}$ satisfy (23), the corresponding variety of the the Noether symmetry is called the perturbation to the the Noether symmetry.

Equation (23) is the determining equation of the perturbation to the the Noether symmetry of the discrete difference variational Hamilton system (1), (2) and (3).

Theorem 2. For the discrete difference variational Hamilton system (1), (2) and (3) perturbed by small quantity $\varepsilon W_{\mathrm{d}}\left(\varphi_{k}\right)$, if the infinitesimal generators $\tau_{k}^{m}, \xi_{k}^{m}$ and $\eta_{k}^{m}$, and discrete gauge function $G_{N k}^{m}$ satisfy the determining of Eq. (23), the perturbation to the the Noether symmetry of the system can induce a $z$-th order discrete adiabatic invariant

$$
\begin{aligned}
& I_{\mathrm{Nz}, \mathrm{d}}=\varepsilon^{m}\left\{\tau_{k}^{m}\left(t_{k}-t_{k-1}\right) D_{2}\left[R_{-} H_{\mathrm{d}}\left(\varphi_{k}\right)\right]\right. \\
& \quad+\xi_{k}^{m}\left(t_{k}-t_{k-1}\right) D_{4}\left[R_{-} H_{\mathrm{d}}\left(\varphi_{k}\right)\right] \\
& \quad+\eta_{k}^{m}\left(t_{k}-t_{k-1}\right) D_{6}\left[R_{-} H_{\mathrm{d}}\left(\varphi_{k}\right)\right]+\tau_{k}^{m} R_{-} H_{\mathrm{d}}\left(\varphi_{k}\right) \\
& \left.\quad-\frac{1}{2}\left(p_{k-1}+p_{k}\right) \xi_{k}^{m}+\frac{1}{2}\left(q_{k-1}+q_{k}\right) \eta_{k}^{m}+G_{N k}^{m}\right\} \\
& \quad(m=0,1, \ldots, z) .
\end{aligned}
$$

Proof: Using representations (7), (8) and (22), the expansion of (23) gives

$$
\begin{aligned}
& H_{\mathrm{d}}\left(\varphi_{k}\right) D_{\mathrm{d}}\left(\tau_{k}^{m}\right)+X_{\mathrm{m}, \mathrm{d}}^{(1)}\left[H_{\mathrm{d}}\left(\varphi_{k}\right)\right] \\
& \quad-\frac{1}{2}\left(p_{k+1}+p_{k}\right) D_{\mathrm{d}}\left(\xi_{k}^{m}\right)+\frac{1}{2}\left(q_{k+1}+q_{k}\right) D_{\mathrm{d}}\left(\eta_{k}^{m}\right) \\
& \quad-W_{\mathrm{d}}\left(\varphi_{k}\right)\left[\xi_{k}^{m-1}-D_{\mathrm{d}}\left(q_{k}\right) \tau_{k}^{m-1}\right]+D_{\mathrm{d}}\left(G_{N k}^{m}\right) \\
& \quad=H_{\mathrm{d}}\left(\varphi_{k}\right) \frac{\tau_{k+1}^{m}-\tau_{k}^{m}}{t_{k+1}-t_{k}}+\tau_{k}^{m} \frac{\partial H_{\mathrm{d}}\left(\varphi_{k}\right)}{\partial t_{k}}+\tau_{k+1}^{m} \frac{\partial H_{\mathrm{d}}\left(\varphi_{k}\right)}{\partial t_{k+1}}
\end{aligned}
$$




$$
\begin{array}{ll}
+\xi_{k}^{m} \frac{\partial H_{\mathrm{d}}\left(\varphi_{k}\right)}{\partial q_{k}}+\xi_{k+1}^{m} \frac{\partial H_{\mathrm{d}}\left(\varphi_{k}\right)}{\partial q_{k+1}}+\eta_{k}^{m} \frac{\partial H_{\mathrm{d}}\left(\varphi_{k}\right)}{\partial p_{k}} & +\eta_{k}^{m}\left[\frac{\partial H_{\mathrm{d}}\left(\varphi_{k}\right)}{\partial p_{k}}+\frac{t_{k}-t_{k-1}}{t_{k+1}-t_{k}} \frac{\partial H_{\mathrm{d}}\left(\varphi_{k-1}\right)}{\partial p_{k}}\right. \\
+\eta_{k+1}^{m} \frac{\partial H_{\mathrm{d}}\left(\varphi_{k}\right)}{\partial p_{k+1}}-\frac{1}{2}\left(p_{k+1}+p_{k}\right) \frac{\xi_{k+1}^{m}-\xi_{k}^{m}}{t_{k+1}-t_{k}} & \left.-\frac{1}{2} \frac{q_{k+1}}{t_{k+1}-t_{k}}+\frac{1}{2} \frac{q_{k-1}}{t_{k+1}-t_{k}}\right] \\
+\frac{1}{2}\left(q_{k+1}+q_{k}\right) \frac{\eta_{k+1}^{m}-\eta_{k}^{m}}{t_{k+1}-t_{k}} & +D_{\mathrm{d}}\left[\tau_{k}^{m}\left(t_{k}-t_{k-1}\right) \frac{\partial H_{\mathrm{d}}\left(\varphi_{k-1}\right)}{\partial t_{k}}\right. \\
-W_{\mathrm{d}}\left(\varphi_{k}\right)\left[\xi_{k}^{m-1}-D_{\mathrm{d}}\left(q_{k}\right) \tau_{k}^{m-1}\right]+D_{\mathrm{d}}\left(G_{N k}^{m}\right) & +\xi_{k}^{m}\left(t_{k}-t_{k-1}\right) \frac{\partial H_{\mathrm{d}}\left(\varphi_{k-1}\right)}{\partial q_{k}} \\
=\tau_{k}^{m} \frac{\partial H_{\mathrm{d}}\left(\varphi_{k}\right)}{\partial t_{k}}+\tau_{k}^{m} \frac{t_{k}-t_{k-1}}{t_{k+1}-t_{k}} \frac{\partial H_{\mathrm{d}}\left(\varphi_{k-1}\right)}{\partial t_{k}} & +\eta_{k}^{m}\left(t_{k}-t_{k-1}\right) \frac{\partial H_{\mathrm{d}}\left(\varphi_{k-1}\right)}{\partial p_{k}} \\
+\tau_{k}^{m} \frac{H_{\mathrm{d}}\left(\varphi_{k-1}\right)}{t_{k+1}-t_{k}}-\tau_{k}^{m} \frac{H_{\mathrm{d}}\left(\varphi_{k}\right)}{t_{k+1}-t_{k}} & +\tau_{k}^{m} H_{\mathrm{d}}\left(\varphi_{k-1}\right)-\frac{1}{2}\left(p_{k-1}+p_{k}\right) \xi_{k}^{m} \\
+\xi_{k}^{m} \frac{\partial H_{\mathrm{d}}\left(\varphi_{k}\right)}{\partial q_{k}}+\xi_{k}^{m} \frac{t_{k}-t_{k-1}}{t_{k+1}-t_{k}} \frac{\partial H_{\mathrm{d}}\left(\varphi_{k-1}\right)}{\partial q_{k}} & \left.+\frac{1}{2}\left(q_{k-1}+q_{k}\right) \eta_{k}^{m}+G_{N k}^{m}\right] \\
-\frac{1}{2} \frac{p_{k-1} \xi_{k}^{m}}{t_{k+1}-t_{k}}+\frac{1}{2} \frac{p_{k+1} \xi_{k}^{m}}{t_{k+1}-t_{k}} & -W_{\mathrm{d}}\left(\varphi_{k}\right)\left[\xi_{k}^{m-1}-D_{\mathrm{d}}\left(q_{k}\right) \tau_{k}^{m-1}\right]=0
\end{array}
$$$$
+\eta_{k}^{m} \frac{\partial H_{\mathrm{d}}\left(\varphi_{k}\right)}{\partial p_{k}}+\eta_{k}^{m} \frac{t_{k}-t_{k-1}}{t_{k+1}-t_{k}} \frac{\partial H_{\mathrm{d}}\left(\varphi_{k-1}\right)}{\partial p_{k}}
$$$$
-\frac{1}{2} \frac{q_{k+1} \eta_{k}^{m}}{t_{k+1}-t_{k}}+\frac{1}{2} \frac{q_{k-1} \eta_{k}^{m}}{t_{k+1}-t_{k}}
$$$$
+\tau_{k+1}^{m} \frac{\partial H_{\mathrm{d}}\left(\varphi_{k}\right)}{\partial t_{k+1}}-\tau_{k}^{m} \frac{t_{k}-t_{k-1}}{t_{k+1}-t_{k}} \frac{\partial H_{\mathrm{d}}\left(\varphi_{k-1}\right)}{\partial t_{k}}
$$$$
+\xi_{k+1}^{m} \frac{\partial H_{\mathrm{d}}\left(\varphi_{k}\right)}{\partial q_{k+1}}-\xi_{k}^{m} \frac{t_{k}-t_{k-1}}{t_{k+1}-t_{k}} \frac{\partial H_{\mathrm{d}}\left(\varphi_{k-1}\right)}{\partial q_{k}}
$$$$
+\eta_{k+1}^{m} \frac{\partial H_{\mathrm{d}}\left(\varphi_{k}\right)}{\partial p_{k+1}}-\eta_{k}^{m} \frac{t_{k}-t_{k-1}}{t_{k+1}-t_{k}} \frac{\partial H_{\mathrm{d}}\left(\varphi_{k-1}\right)}{\partial p_{k}}
$$$$
+\tau_{k+1}^{m} \frac{H_{\mathrm{d}}\left(\varphi_{k}\right)}{t_{k+1}-t_{k}}-\tau_{k}^{m} \frac{H_{\mathrm{d}}\left(\varphi_{k-1}\right)}{t_{k+1}-t_{k}}
$$$$
+\frac{1}{2} \frac{p_{k-1} \xi_{k}^{m}}{t_{k+1}-t_{k}}-\frac{1}{2} \frac{p_{k+1} \xi_{k+1}^{m}}{t_{k+1}-t_{k}}-\frac{1}{2} \frac{p_{k} \xi_{k+1}^{m}}{t_{k+1}-t_{k}}
$$$$
+\frac{1}{2} \frac{p_{k} \xi_{k}^{m}}{t_{k+1}-t_{k}}-\frac{1}{2} \frac{q_{k-1} \eta_{k}^{m}}{t_{k+1}-t_{k}}+\frac{1}{2} \frac{q_{k+1} \eta_{k+1}^{m}}{t_{k+1}-t_{k}}
$$$$
+\frac{1}{2} \frac{q_{k} \eta_{k+1}^{m}}{t_{k+1}-t_{k}}-\frac{1}{2} \frac{q_{k} \eta_{k}^{m}}{t_{k+1}-t_{k}}+D_{\mathrm{d}}\left(G_{N k}^{m}\right)
$$$$
-W_{\mathrm{d}}\left(\varphi_{k}\right)\left[\xi_{k}^{m-1}-D_{\mathrm{d}}\left(q_{k}\right) \tau_{k}^{m-1}\right]
$$$$
=\tau_{k}^{m}\left[\frac{\partial H_{\mathrm{d}}\left(\varphi_{k}\right)}{\partial t_{k}}+\frac{t_{k}-t_{k-1}}{t_{k+1}-t_{k}} \frac{\partial H_{\mathrm{d}}\left(\varphi_{k-1}\right)}{\partial t_{k}}\right.
$$$$
\left.+\frac{H_{\mathrm{d}}\left(\varphi_{k-1}\right)}{t_{k+1}-t_{k}}-\frac{H_{\mathrm{d}}\left(\varphi_{k}\right)}{t_{k+1}-t_{k}}\right]
$$$$
+\xi_{k}^{m}\left[\frac{\partial H_{\mathrm{d}}\left(\varphi_{k}\right)}{\partial q_{k}}+\frac{t_{k}-t_{k-1}}{t_{k+1}-t_{k}} \frac{\partial H_{\mathrm{d}}\left(\varphi_{k-1}\right)}{\partial q_{k}}\right.
$$$$
\left.-\frac{1}{2} \frac{p_{k-1}}{t_{k+1}-t_{k}}+\frac{1}{2} \frac{p_{k+1}}{t_{k+1}-t_{k}}\right]
$$

Using (13)-(15), from (25), we obtain

$$
\begin{aligned}
D_{\mathrm{d}} & \left\{\varepsilon ^ { m } \left[\tau_{k}^{m}\left(t_{k}-t_{k-1}\right) \frac{\partial H_{\mathrm{d}}\left(\varphi_{k-1}\right)}{\partial t_{k}}\right.\right. \\
& +\xi_{k}^{m}\left(t_{k}-t_{k-1}\right) \frac{\partial H_{\mathrm{d}}\left(\varphi_{k-1}\right)}{\partial q_{k}} \\
& +\eta_{k}^{m}\left(t_{k}-t_{k-1}\right) \frac{\partial H_{\mathrm{d}}\left(\varphi_{k-1}\right)}{\partial p_{k}}+\tau_{k}^{m} H_{\mathrm{d}}\left(\varphi_{k-1}\right) \\
& \left.\left.-\frac{1}{2}\left(p_{k-1}+p_{k}\right) \xi_{k}^{m}+\frac{1}{2}\left(q_{k-1}+q_{k}\right) \eta_{k}^{m}+G_{N k}^{m}\right]\right\} \\
& =D_{\mathrm{d}}\left\{\varepsilon ^ { m } \left[\tau_{k}^{m}\left(t_{k}-t_{k-1}\right) D_{2}\left(R_{-} H_{\mathrm{d}}\left(\varphi_{k}\right)\right)\right.\right. \\
& +\xi_{k}^{m}\left(t_{k}-t_{k-1}\right) D_{4}\left(R_{-} H_{\mathrm{d}}\left(\varphi_{k}\right)\right) \\
& +\eta_{k}^{m}\left(t_{k}-t_{k-1}\right) D_{6}\left(R_{-} H_{\mathrm{d}}\left(\varphi_{k}\right)\right)+\tau_{k}^{m} R_{-} H_{\mathrm{d}}\left(\varphi_{k}\right) \\
& \left.\left.-\frac{1}{2}\left(p_{k-1}+p_{k}\right) \xi_{k}^{m}+\frac{1}{2}\left(q_{k-1}+q_{k}\right) \eta_{k}^{m}+G_{N k}^{m}\right]\right\} \\
& =D_{\mathrm{d}} I_{\mathrm{Nz}, \mathrm{d}} \\
& =\varepsilon^{m}\left\{\varepsilon W_{\mathrm{d}}\left(\varphi_{k}\right) \frac{q_{k+1}-q_{k}}{t_{k+1}-t_{k}} \tau_{k}^{m}-\varepsilon W_{\mathrm{d}}\left(\varphi_{k}\right) \xi_{k}^{m}\right. \\
& \left.+W_{\mathrm{d}}\left(\varphi_{k}\right)\left[\xi_{k}^{m-1}-D_{\mathrm{d}}\left(q_{k}\right) \tau_{k}^{m-1}\right]\right\} \\
& =\varepsilon^{m}\left\{-\varepsilon W_{\mathrm{d}}\left(\varphi_{k}\right)\left[\xi_{k}^{m}-D_{\mathrm{d}}\left(q_{k}\right) \tau_{k}^{m}\right]\right. \\
& \left.+W_{\mathrm{d}}\left(\varphi_{k}\right)\left[\xi_{k}^{m-1}-D_{\mathrm{d}}\left(q_{k}\right) \tau_{k}^{m-1}\right]\right\} \\
& =-\varepsilon^{z+1} W_{\mathrm{d}}\left(\varphi_{k}\right)\left[\xi_{k}^{z}-D_{\mathrm{d}}\left(q_{k}\right) \tau_{k}^{z}\right] .
\end{aligned}
$$

It shows that $D_{\mathrm{d}} I_{\mathrm{Nz}, \mathrm{d}}$ is directly proportional to $\varepsilon^{z+1}$, so $I_{\mathrm{Nz}, \mathrm{d}}$ is a $z$-th order adiabatic invariant of the discrete difference variational Hamilton system, which can be called the discrete the Noether adiabatic invariant. When $z=0$, which mean that $\varepsilon W_{\mathrm{d}}\left(\varphi_{k}\right)$ vanish, the system becomes the one without perturbation. Then the 
discrete the Noether adiabatic invariant (24) turns into the discrete the Noether exact invariant (10).

\section{An example}

Take the Emden equation to illustrate the application of the above results. The discrete Hamiltonian of the system and its recursive operator are

$$
\begin{aligned}
& H_{\mathrm{d}}\left(\varphi_{k}\right)=\frac{1}{2} \frac{p_{k+1}^{2}+p_{k}^{2}}{t_{k+1}^{2}+t_{k}^{2}}+\frac{1}{12}\left(t_{k+1}^{2} q_{k+1}^{6}+t_{k}^{2} q_{k}^{6}\right), \\
& R_{-} H_{\mathrm{d}}\left(\varphi_{k}\right)=H_{\mathrm{d}}\left(\varphi_{k-1}\right)=\frac{1}{2} \frac{p_{k}^{2}+p_{k-1}^{2}}{t_{k}^{2}+t_{k-1}^{2}} \\
& \quad+\frac{1}{12}\left(t_{k}^{2} q_{k}^{6}+t_{k-1}^{2} q_{k-1}^{6}\right),
\end{aligned}
$$

and the system is perturbed by the small quantity

$$
\varepsilon W_{\mathrm{d}}\left(\varphi_{k}\right)=-\varepsilon \frac{q_{k+1} p_{k+1}-q_{k} p_{k}}{3 q_{k} t_{k}-q_{k} t_{k+1}-2 q_{k+1} t_{k}} .
$$

Let us study the perturbation to the the Noether symmetry and the the Noether adiabatic invariant of the discrete difference variational Hamilton system.

We calculate the $D_{j} H_{\mathrm{d}}\left(\varphi_{k}\right)(j=1,2,3,4,5,6)$ as

$$
\begin{aligned}
& D_{1} H_{\mathrm{d}}\left(\varphi_{k}\right)=-\frac{t_{k}\left(p_{k+1}^{2}+p_{k}^{2}\right)}{\left(t_{k+1}^{2}+t_{k}^{2}\right)^{2}}+\frac{1}{6} t_{k} q_{k}^{6}, \\
& D_{2} H_{\mathrm{d}}\left(\varphi_{k}\right)=-\frac{t_{k+1}\left(p_{k+1}^{2}+p_{k}^{2}\right)}{\left(t_{k+1}^{2}+t_{k}^{2}\right)^{2}}+\frac{1}{6} t_{k+1} q_{k+1}^{6}, \\
& D_{3} H_{\mathrm{d}}\left(\varphi_{k}\right)=\frac{1}{2} t_{k}^{2} q_{k}^{5}, \quad D_{4} H_{\mathrm{d}}\left(\varphi_{k}\right)=\frac{1}{2} t_{k+1}^{2} q_{k+1}^{5}, \\
& D_{5} H_{\mathrm{d}}\left(\varphi_{k}\right)=\frac{p_{k}}{t_{k+1}^{2}+t_{k}^{2}}, \quad D_{6} H_{\mathrm{d}}\left(\varphi_{k}\right)=\frac{p_{k+1}}{t_{k+1}^{2}+t_{k}^{2}} .
\end{aligned}
$$

Firstly, we seek the discrete the Noether exact invariant. Suppose that the generators $\tau_{k}^{0}, \xi_{k}^{0}$ and $\eta_{k}^{0}$ are linear, i.e.

$$
\begin{aligned}
& \tau_{k}^{0}\left(t_{k}, q_{k}, p_{k}\right)=C_{1} t_{k}+C_{2} q_{k}+C_{3} p_{k}+C_{4}, \\
& \xi_{k}^{0}\left(t_{k}, q_{k}, p_{k}\right)=C_{5} t_{k}+C_{6} q_{k}+C_{7} p_{k}+C_{8}, \\
& \eta_{k}^{0}\left(t_{k}, q_{k}, p_{k}\right)=C_{9} t_{k}+C_{10} q_{k}+C_{11} p_{k}+C_{12},
\end{aligned}
$$

where $C_{1}-C_{12}$ are constants.

Substituting (30) and (31) into the the Noether identity (9) of the system without perturbation, we have

$$
\begin{aligned}
& {\left[\frac{1}{2} \frac{p_{k+1}^{2}+p_{k}^{2}}{t_{k+1}^{2}+t_{k}^{2}}+\frac{1}{12}\left(t_{k+1}^{2} q_{k+1}^{6}+t_{k}^{2} q_{k}^{6}\right)\right] D_{\mathrm{d}}\left(\tau_{k}^{0}\right)} \\
& \quad+\left[\frac{1}{6} t_{k} q_{k}^{6}-\frac{t_{k}\left(p_{k+1}^{2}+p_{k}^{2}\right)}{\left(t_{k+1}^{2}+t_{k}^{2}\right)^{2}}\right] \tau_{k}^{0} \\
& \quad+\left[\frac{1}{6} t_{k+1} q_{k+1}^{6}-\frac{t_{k+1}\left(p_{k+1}^{2}+p_{k}^{2}\right)}{\left(t_{k+1}^{2}+t_{k}^{2}\right)^{2}}\right] \tau_{k+1}^{0} \\
& \quad+\frac{1}{2} t_{k}^{2} q_{k}^{5} \xi_{k}^{0}+\frac{1}{2} t_{k+1}^{2} q_{k+1}^{5} \xi_{k+1}^{0}+\frac{p_{k} \eta_{k}^{0}}{t_{k+1}^{2}+t_{k}^{2}} \\
& \quad+\frac{p_{k+1} \eta_{k+1}^{0}}{t_{k+1}^{2}+t_{k}^{2}}-\frac{1}{2}\left(p_{k+1}+p_{k}\right) D_{\mathrm{d}}\left(\xi_{k}^{0}\right)
\end{aligned}
$$

$$
+\frac{1}{2}\left(q_{k+1}+q_{k}\right) D_{\mathrm{d}}\left(\eta_{k}^{0}\right)+D_{\mathrm{d}}\left(G_{N k}^{0}\right)=0 .
$$

It follows that when

$$
\begin{aligned}
& \tau_{k}^{0}\left(t_{k}, q_{k}, p_{k}\right)=2 t_{k}, \quad \xi_{k}^{0}\left(t_{k}, q_{k}, p_{k}\right)=-q_{k}, \\
& \eta_{k}^{0}\left(t_{k}, q_{k}, p_{k}\right)=p_{k},
\end{aligned}
$$

the function

$$
G_{N k}^{0}=-q_{k} p_{k}
$$

satisfies the the Noether identity (9). According to Theorem 1 , the discrete difference variational Hamilton system has the following discrete the Noether exact invariant:

$$
\begin{aligned}
& I_{\mathrm{N} 0, \mathrm{~d}}=\frac{t_{k}\left(p_{k}^{2}+p_{k-1}^{2}\right)}{t_{k}^{2}+t_{k-1}^{2}}-\frac{2 t_{k}^{2}\left(t_{k}-t_{k-1}\right)\left(p_{k}^{2}+p_{k-1}^{2}\right)}{\left(t_{k}^{2}+t_{k-1}^{2}\right)^{2}} \\
& +\frac{\left(t_{k}-t_{k-1}\right) p_{k}^{2}}{t_{k}^{2}+t_{k-1}^{2}}+\frac{1}{6} t_{k} t_{k-1}\left(t_{k} q_{k}^{6}+t_{k-1} q_{k-1}^{6}\right) \\
& +\frac{1}{2}\left(p_{k-1} q_{k}+p_{k} q_{k-1}\right)=\mathrm{const} .
\end{aligned}
$$

Secondly, we seek the first order discrete the Noether adiabatic invariant. We also suppose that the generators $\tau_{k}^{1}, \xi_{k}^{1}$ and $\eta_{k}^{1}$ are linear, i.e.

$$
\begin{aligned}
& \tau_{k}^{1}\left(t_{k}, q_{k}, p_{k}\right)=C_{1}^{\prime} t_{k}+C_{2}^{\prime} q_{k}+C_{3}^{\prime} p_{k}+C_{4}^{\prime}, \\
& \xi_{k}^{1}\left(t_{k}, q_{k}, p_{k}\right)=C_{5}^{\prime} t_{k}+C_{6}^{\prime} q_{k}+C_{7}^{\prime} p_{k}+C_{8}^{\prime}, \\
& \eta_{k}^{1}\left(t_{k}, q_{k}, p_{k}\right)=C_{9}^{\prime} t_{k}+C_{10}^{\prime} q_{k}+C_{11}^{\prime} p_{k}+C_{12}^{\prime},
\end{aligned}
$$

where $C_{1}^{\prime}-C_{12}^{\prime}$ are constants.

Substituting (30), (31) and (36) into the determining Eq. (23) of the perturbation to the the Noether symmetry of the system, we have

$$
\begin{aligned}
& {\left[\frac{1}{2} \frac{p_{k+1}^{2}+p_{k}^{2}}{t_{k+1}^{2}+t_{k}^{2}}+\frac{1}{12}\left(t_{k+1}^{2} q_{k+1}^{6}+t_{k}^{2} q_{k}^{6}\right)\right] D_{\mathrm{d}}\left(\tau_{k}^{1}\right)} \\
& \quad+\left[\frac{1}{6} t_{k} q_{k}^{6}-\frac{t_{k}\left(p_{k+1}^{2}+p_{k}^{2}\right)}{\left(t_{k+1}^{2}+t_{k}^{2}\right)^{2}}\right] \tau_{k}^{1} \\
& \quad+\left[\frac{1}{6} t_{k+1} q_{k+1}^{6}-\frac{t_{k+1}\left(p_{k+1}^{2}+p_{k}^{2}\right)}{\left(t_{k+1}^{2}+t_{k}^{2}\right)^{2}}\right] \tau_{k+1}^{1}+\frac{1}{2} t_{k}^{2} q_{k}^{5} \xi_{k}^{1} \\
& \quad+\frac{1}{2} t_{k+1}^{2} q_{k+1}^{5} \xi_{k+1}^{1}+\frac{p_{k} \eta_{k}^{1}}{t_{k+1}^{2}+t_{k}^{2}}+\frac{p_{k+1} \eta_{k+1}^{1}}{t_{k+1}^{2}+t_{k}^{2}} \\
& \quad-\frac{1}{2}\left(p_{k+1}+p_{k}\right) D_{\mathrm{d}}\left(\xi_{k}^{1}\right)+\frac{1}{2}\left(q_{k+1}+q_{k}\right) D_{\mathrm{d}}\left(\eta_{k}^{1}\right) \\
& \quad+\frac{q_{k+1} p_{k+1}-q_{k} p_{k}}{3 q_{k} t_{k}-q_{k} t_{k+1}-2 q_{k+1} t_{k}}\left[\xi_{k}^{0}-D_{\mathrm{d}}\left(q_{k}\right) \tau_{k}^{0}\right] \\
& \quad+D_{\mathrm{d}}\left(G_{N k}^{1}\right)=0 .
\end{aligned}
$$

It follows that when

$$
\begin{gathered}
\tau_{k}^{1}\left(t_{k}, q_{k}, p_{k}\right)=2 t_{k}, \quad \xi_{k}^{1}\left(t_{k}, q_{k}, p_{k}\right)=-q_{k}, \\
\eta_{k}^{1}\left(t_{k}, q_{k}, p_{k}\right)=p_{k},
\end{gathered}
$$

the function

$$
G_{N k}^{1}=-2 q_{k} p_{k}
$$

satisfies the determining Eq. (23) of the perturbation to 
the Noether symmetry. According to Theorem 2, the discrete difference variational Hamilton system has the following first order discrete the Noether adiabatic invariant:

$$
\begin{gathered}
I_{\mathrm{N} 1, \mathrm{~d}}=\left[\frac{t_{k}\left(p_{k}^{2}+p_{k-1}^{2}\right)}{t_{k}^{2}+t_{k-1}^{2}}-\frac{2 t_{k}^{2}\left(t_{k}-t_{k-1}\right)\left(p_{k}^{2}+p_{k-1}^{2}\right)}{\left(t_{k}^{2}+t_{k-1}^{2}\right)^{2}}\right. \\
+\frac{\left(t_{k}-t_{k-1}\right) p_{k}^{2}}{t_{k}^{2}+t_{k-1}^{2}}+\frac{1}{6} t_{k} t_{k-1}\left(t_{k} q_{k}^{6}+t_{k-1} q_{k-1}^{6}\right) \\
\left.+\frac{1}{2}\left(p_{k-1} q_{k}+p_{k} q_{k-1}\right)\right](1+\varepsilon)-\varepsilon q_{k} p_{k} .
\end{gathered}
$$

Further we can obtain more high-order discrete adiabatic invariants. In addition, it is worth noting that there are many calculations above, which can be also done in an automatic way using a computer algebra system stated in Refs. [26, 27].

\section{Conclusion}

The perturbation to the the Noether symmetry and the the Noether adiabatic invariants of discrete difference variational systems are studied in this paper. We obtain the discrete the Noether adiabatic invariant induced directly by the perturbation to the Noether symmetry of the system. When $z=0$, this means that $\varepsilon W_{\mathrm{d}}\left(\varphi_{k}\right)$ vanish, the system becomes the one without perturbations, and the discrete the Noether adiabatic invariant will turn into the discrete the Noether exact invariant (i.e. the so-called discrete the Noether conserved quantity in Ref. [14]) naturally. The results of this paper have important significance for further study on the discrete mechanical systems and symmetrical perturbation theory.

\section{Acknowledgments}

This research was supported by the National Natural Science Foundation of China (grant No. 11103086) and Fundamental Research Funds for the Central Universities of China (grant No. 09CX04018A).

\section{References}

[1] F.X. Mei, Symmetries and Conserved Quantities of Constrained Mechanical Systems, Beijing Institute of Technology Press, Beijing 2004 (in Chinese).

[2] S.K. Luo, Y.F. Zhang, et al., Advances in the Study of Dynamics of Constrained Mechanics Systems, Science Press, Beijing 2008 (in Chinese).
[3] D.F.M. Torres, in: Dynamics, Bifurcations, and Control, Kloster Irsee, 2001, Lecture Notes in Control and Inform. Sci., Eds. F. Colonius, L. Grüne, Vol. 273, Springer, Berlin 2002, p. 287.

[4] D.F.M. Torres, Eur. J. Control. 8, 56 (2002).

[5] D.F.M. Torres, Commun. Pure Appl. Anal. 3, 491 (2004).

[6] D. Levi, R. Yamilov, J. Math. Phys. 38, 6648 (1997).

[7] D. Levi, S. Tremblay, P. Winternitz, J. Phys. A, Math. Gen. 33, 8507 (2000).

[8] D. Levi, S. Tremblay, P. Winternitz, J. Phys. A, Math. Gen. 34, 9507 (2001).

[9] V. Dorodnitsyn, Appl. Numer. Math. 39, 307 (2001).

[10] Z. Bartosiewicz, D.F.M. Torres, J. Math. Anal. Appl. 342, 1220 (2008).

[11] N. Martins, D.F.M. Torres, Appl. Math. Lett. 23, 1432 (2010).

[12] Z. Bartosiewicz, N. Martins, D.F.M. Torres, Eur. J. Control. 17, 9 (2011).

[13] S.Y. Shi, J.L. Fu, L.Q. Chen, Chin. Phys. B 17, 385 (2008).

[14] S.Y. Shi, X.H. Huang, X.B. Zhang, L. Jin, Acta Phys. Sin. 58, 3625 (2009) (in Chinese).

[15] J.L. Fu, H. Fu, R.W. Liu, Phys. Lett. A 374, 1812 (2010).

[16] J.M. Burgers, Ann. Phys. 357, 195 (1917).

[17] Y.Y. Zhao, F.X. Mei, Symmetries and Invariants of Mechanical Systems, Science Press, Beijing 1999 (in Chinese)

[18] J.L. Fu, L.Q. Chen, Phys. Lett. A 324, 95 (2004).

[19] X.W. Chen, Y.M. Li, Y.H. Zhao, Phys. Lett. A 337, 274 (2005).

[20] M.J. Zhang, J.H. Fang, X.N. Zhang, K. Lu, Chin. Phys. B 17, 1957 (2008).

[21] N. Ding, X.F. Chen, J.H. Fang, C.Z. Liu, Phys. Lett. A 373, 3005 (2009).

[22] M.J. Zhang, J.H. Fang, K. Lu, T. Pang, P. Lin, Commun. Theor. Phys. 51, 961 (2009).

[23] M.J. Zhang, J.H. Fang, K. Lu, Int. J. Theor. Phys. 49, 427 (2010).

[24] M.J. Zhang, J.H. Fang, K. Lu, K.J. Zhang, Y. Li, Chin. Phys. Lett. 26, 120201 (2009).

[25] P. Wang, H.J. Zhu, Acta Phys. Pol. A 119, 298 (2011).

[26] P.D.F. Gouveia, D.F.M. Torres, E.A.M. Rocha, Control Cybernet. 35, 831 (2006).

[27] P.D.F. Gouveia, D.F.M. Torres, Nonlinear Anal. 71, e138 (2009). 